\title{
Research on the Traction and Obstacle-Surmounting Performance of an Adaptive Pipeline-Plugging Robot
}

\author{
Hongwei Yan ${ }^{1, *}$ - Jian $\mathrm{Li}^{1}$ - Ziming Kou ${ }^{2}-\mathrm{Yi} \mathrm{Liu}^{1}$ - Pengcheng $\mathrm{Li}^{1}-\mathrm{Lu}$ Wang1 \\ ${ }^{1}$ North University of China, School of Mechanical Engineering, China \\ ${ }^{2}$ Taiyuan University of Technology, College of Mechanical and Transport Engineering, China
}

To solve a series of problems, such as damage, increased impurities and obstacles caused by medium corrosion, and erosion in the long-term use of the pipeline, a multi-body distributed adaptive pipeline plugging and repairing robot with good driving performance and strong reliability is proposed by using the modular design method. By establishing the obstacle-crossing model of the robot, the traction equation and obstaclecrossing equation of the driving wheel and supporting wheel are studied. It can be seen from the equation that reducing the deflection angle of the driving wheel or reducing the speed of the motor spindle can improve the obstacle-crossing ability. The driving unit model is established in ADAMS software, and its running speed, driving force, and obstacle-crossing height are simulated and analysed. The model prototype is established and verified with experiments. When the deflection angle of the robot driving wheel is set to $20^{\circ}$ to $35^{\circ}$, it meets the design requirements. When the deflection angle of the driving wheel is set to $20^{\circ}$, the robot has large traction, good running stability and high obstacle-crossing height. The maximum obstacle-crossing height is $6 \mathrm{~mm}$. At this time, the robot reaches the best running state. The design of an adaptive leakage plugging robot for buried pipelines provides an important reference for the research and development of pipeline emergency prevention and control equipment.

Keywords: pipeline leakage, plugging robot, obstacle crossing, optimum driving angle, simulation analysis

\begin{abstract}
Highlights
- A pipeline leakage self-adaptive sealing robot was designed to realize pipeline leakage detection, rapid plugging and repair, and stable and reliable operation.

- This robot has a certain degree of self-adaptive ability, sufficient driving force, better blocking ability, and good obstacle-crossing ability.

- The optimum deflection angle of the driving wheel is obtained by analysis. The influence of the change of deflection angle on the obstacle-crossing performance of the robot. Under the optimal deflection angle, the pipeline robot runs smoothly and has a good ability to pass over obstacles.
\end{abstract}

\section{INTRODUCTION}

As the fifth-largest transportation method, pipeline transportation has the advantages of low cost, safety, and reliability and is widely used [1] to [3]. With the large-scale use of pipelines and the increase in their service time, they can be affected by internal corrosion and human intervention, and thus pipelines will always be prone to rupture and erosion [4] to [6]. Buried pipelines will transport oil and natural gas to avoid the interference of external factors and save space [7] to [9]. When there is a leak in a buried pipeline, it is usually excavated directly to find the leakage point and then treated by sectional plugging and repair [10] and [11]. Both pipe wells and buried pipelines encounter difficulties locating leaks and a working environment that is too small, which is not conducive to manual operation [12] to [14]. Therefore, this paper proposes a multi-body distributed pipeline leak self-adaptive plugging robot composed of a pipeline robot and a leak-plugging unit. The driving unit provides a dynamic for the adaptive pipelineplugging robot to detect the location of pipeline leaks.
The leak-plugging unit seals and repairs the pipeline leaks. Numerous in-depth studies have examined this problem. In terms of pipeline robots, the Brazilian CLIDADE team designed an oil and gas pressuredriven robot based on the principle of medium pressure difference. It has built-in ultrasonic flaw detection equipment to detect pipeline leaks and leak point sizes [15]. At Shandong University, Han Meng designed a four-wheeled robot that adopts a modular tandem design and is connected by two robots with universal coupling [16] and [17]. Liu of Southwest Petroleum University in China designed a spiraldriven pipeline robot. He reduced the torque of the spindle motor through the bevel gear and acted on the robot driving wheel. Theoretically, he deduced the relationship between the helical inclination of the driving wheel, the load and the moving speed [18] to [20]. The Canadian company Inuktun developed a crawler robot that can smoothly pass through complex pipelines and is suitable for pipelines with more damage or severe corrosion [21] and [22]. Woongsun Jeon et al. in the United States developed a four-link peristaltic robot, in which the front and rear bodies 
can be locked or moved relative to the soft axis, and the relative movement between the two bodies can achieve the purpose of moving in the pipe [23] to [25]. Zagler et al. at the Technical University of Munich, Germany, developed the eight-legged robot MORITZ, which is equipped with precision instruments such as angle sensors, microgravity sensors and five-axis acceleration sensors. Although it has good adaptability, its structure is complex, and control is difficult [26] and [27]. A spiral robot makes use of a driving wheel to create spiral motions on the inner wall of the pipeline and transform the circumferential rotation force into axial forward power. It has the characteristics of a high driving force, stable operation, and a simple control mode and is suitable for running in complex pipelines, which has become the focus of this paper. In terms of leakage and plugging, the Oil-States Hydro Tech company was the first to use a device for plugging pipeline leakage points by external pressure, which used the fixture to compress the pipeline leakage point to achieve the purpose of plugging and repairing, mainly for small single-point leakage [28] and [29]. The in-pipe intelligent plugging mechanism designed by Bowie et al. is an in-pipe repair platform composed of a drive system, a plugging system and a carrying system [30]. The United States FurmanIte company was the first to develop a perforated plugger that integrates perforation, plugging, and transmission, which is widely used in deep-sea highpressure pipeline leakage [31]. Many scholars have studied different pipeline leakage situations and proposed different plugging and repair methods to achieve the effect of rapid plugging and repair, but at present, few combine pipeline robots with plugging and repair devices. In this context, this paper focuses on the single-point leakage, multipoint leakage, and multipoint long-spacing scattered section leakage of diameter $150 \mathrm{~mm}$ buried pipeline, aiming to design a new type of pipeline leakage adaptive plugging robot that integrates in-pipe plugging and quick repair. This article aims to study and analyse the walking stability and obstacle-crossing performance of the robot device, which can lay a theoretical foundation for the in-depth research of a future blockage repair robot.

\section{DESIGN OF A PLUGGING ROBOT STRUCTURE}

\subsection{Overall Structure}

The buried pipeline leak self-adaptive plugging robot is primarily intended for pipeline leakage and plugging operations. The buried pipeline environment is complex; the space is small, and over time, various impurities will accrue in the pipe. Therefore, the designed device must have the following characteristics: operability in a complex environment, a certain degree of self-adaptive ability, sufficient traction and driving force, accurate identification of pipeline leakage points and leakage patterns, good operational stability, good plugging ability, and a defined operating speed.

This article intends to design a pipeline emergency plugging and repairing robot that can work smoothly under various complex working conditions to meet the above requirements. According to the previous research results of pipeline robots, the overall structure design needs to meet the design indicators in Table 1. Since the materials transported by buried pipelines are generally flammable and explosive, such as oil and natural gas, and may be corrosive, the mechanical structure of the buried pipeline leakage self-adaptive plugging robot is designed with a protective shell. The buried pipeline leak self-adaptive plugging robot consists of two self-adaptive plugging robots connected by electromagnetic adsorption modules, which can realize docking and separation according to actual working conditions.

Table 1. Design index of the adaptive plugging robot for buried pipeline leakage

\begin{tabular}{clc}
\hline $\begin{array}{c}\text { Serial } \\
\text { number }\end{array}$ & \multicolumn{2}{c}{ Design indicators } \\
\hline 1 & Pipe diameter fitting range & $\phi 140 \mathrm{~mm}$ to $\phi 160 \mathrm{~mm}$ \\
\hline 2 & $\begin{array}{l}\text { The radius of minimum curvature } \\
\text { that can pass through the bend }\end{array}$ & $p \geq 550 \mathrm{~mm}$ \\
\hline 3 & Plugging pressure range & $0.1 \mathrm{MPa}$ \\
\hline 4 & Walking speed in straight pipe & $0.01 \mathrm{~m} / \mathrm{s}$ to $0.04 \mathrm{~m} / \mathrm{s}$ \\
\hline 5 & Walking speed in curved pipe & $0.005 \mathrm{~m} / \mathrm{s}$ to $0.01 \mathrm{~m} / \mathrm{s}$ \\
\hline 6 & Drive unit mass & $m_{1} \leq 5 \mathrm{~kg}$ \\
\hline 7 & Drive unit length & $L_{1} \leq 300 \mathrm{~mm}$ \\
\hline 8 & System traction & $F \geq 20 \mathrm{~N}$ \\
\hline 9 & Mass of plugging unit & $m_{2} \leq 5 \mathrm{~kg}$ \\
\hline 10 & Plug unit length & $L_{2} \leq 300 \mathrm{~mm}$ \\
\hline
\end{tabular}

The adaptive plugging robot comprises a drive unit, a leak-plugging unit, and a control unit. The driving unit is the power source of the whole system and provides the driving force for the system; the control components of the whole robot are integrated into the control unit box; and the leakage plugging unit is the working unit of the system, which can plug and repair the leakage point. The parallel coupling connects each unit into a whole, and the structure of the self-adaptive plugging robot for buried pipeline leakage is shown in Fig. 1. The pipeline robot can be 


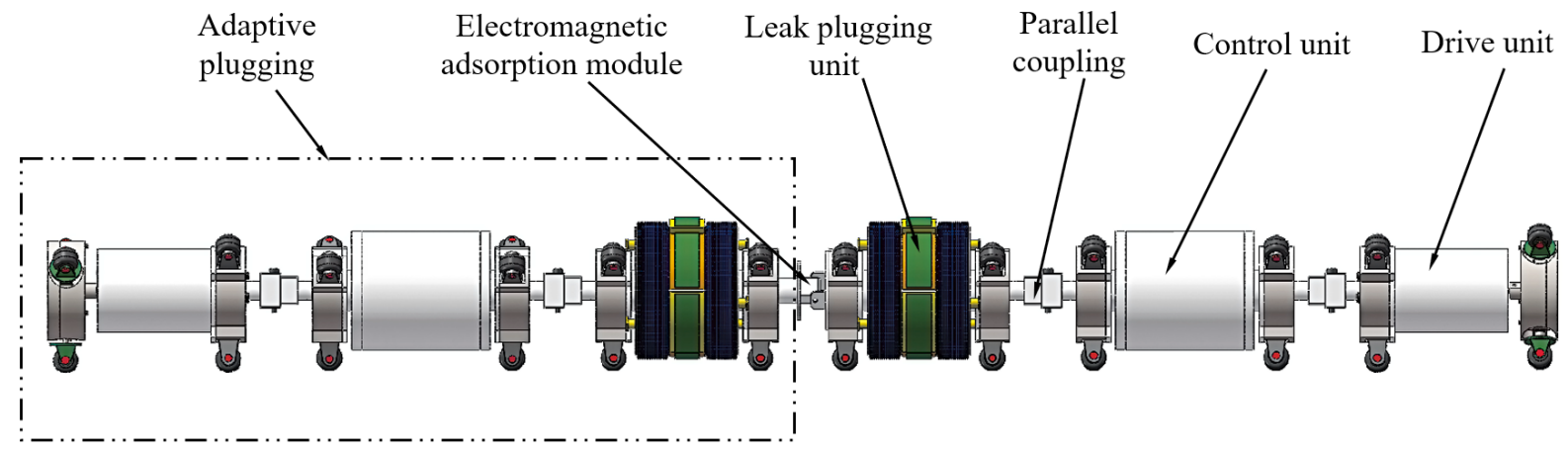

Fig. 1. Structure diagram of a buried pipeline leak self-adaptive plugging robot

applied to pipeline leakage detection and intelligent emergency treatment. In case of leakage, the leakage point can be quickly blocked and repaired, and the uninterrupted oil and gas transmission and leakage port cannot be expanded. Only one is selected for analysis because the main structures of the two adaptive plugging robots are exactly the same.

\subsection{Drive Unit}

The drive unit adopts a closed design, as shown in Fig. 2. It comprises a screwdriver mechanism, a drive motor, and a supporting mechanism. The drive unit adopts a screw-drive as the main transmission mode, and the support mechanism adopts the telescopic spring support. The drive motor provides torque for the screw drive mechanism.

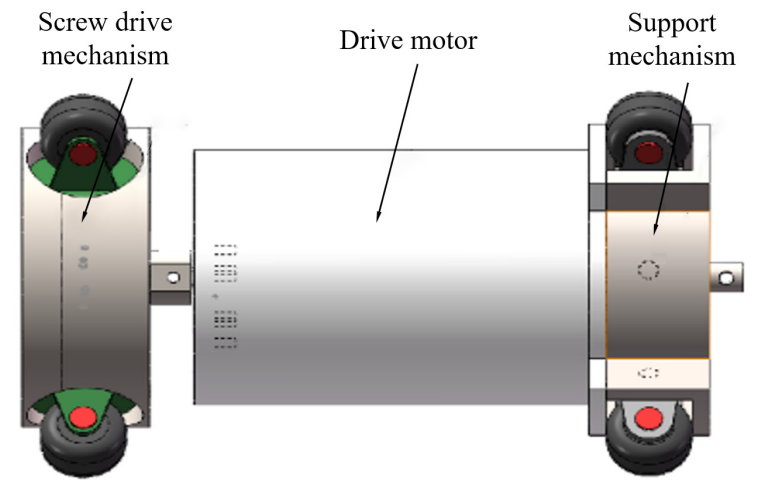

Fig. 2. Drive unit structure diagram

The components of the screw drive mechanism include a fixed shell, a driving wheel, a wheel frame, an angle steering gear, a wheel axle, a spring, a fixed frame, and a protective shell, as shown in Fig. 3.

The shell of the screw drive mechanism is composed of a fixed shell and a protective shell. The fixed frame is connected with an adjustable angle steering gear, a wheel frame, and a driving wheel.
The fixed housing is connected with the drive motor. The fixed shell is connected with the drive motor. When the drive motor rotates, the motor shaft drives the fixed housing to rotate. At the same time, the fixed shell can rotate the angle-adjusting steering gear, wheel carrier and driving wheel. The driving wheel is installed on the wheel frame, and the wheel frame is fixedly connected with the steering gear axis, so the steering gear can be used to control the wheel frame deflection. The wheel frame limits the position of the angle-adjusting steering gear in the drive unit, and the wheel frame is designed to ensure that the angle-adjusting steering gear can only move up and down. A spring is installed on the bottom of the angle-adjusting steering gear, and the spring is used to connect the steering gear to the wheel frame. The spring plays an adjustment role so that the driving wheel and the steering gear can move up and down with the expansion and contraction of the spring, thereby completing the robot's self-control adaptation. The drive motor is the power core of the entire system and provides torque for the drive unit.

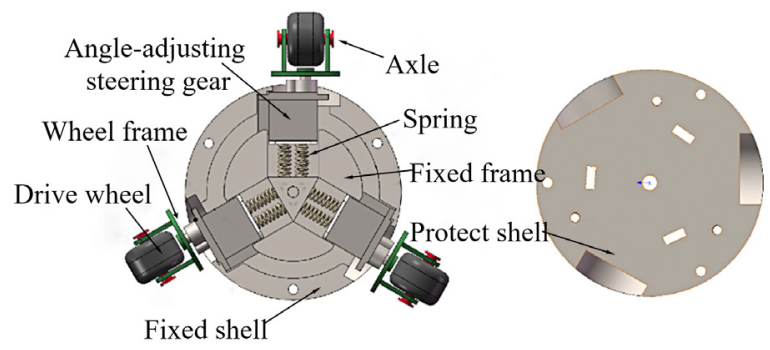

Fig. 3. Structural diagram of the screw drive mechanism

The supporting mechanism is composed of nine parts: a supporting wheel, a supporting wheel frame, an inner shell, a supporting wheel shaft, a connecting rod, a spring fixing shell, a spring, an assembly shell, and an outer shell, as shown in Fig. 4. The outer shell structure of the supporting mechanism is composed 
of an inner shell and an outer shell, which guide the direction of the supporting wheel. The lower end of the connecting rod is connected with a spring, and the upper end is connected with the supporting wheel frame. The spring fixing shell restricts the connecting rod in a circumferential direction so that it can only move up and down. The inner shell is designed with a notch that matches the outer structure of the supporting wheel frame so that the supporting wheel frame can only move up and down. The expansion and contraction of the spring act on the support wheel through the connecting rod so that it can expand and contract within a certain range to enable the functions of support and obstacle crossing. The two parts of the screw drive mechanism and the support mechanism work in coordination so that the entire robot can run adaptively and smoothly in complex pipelines.

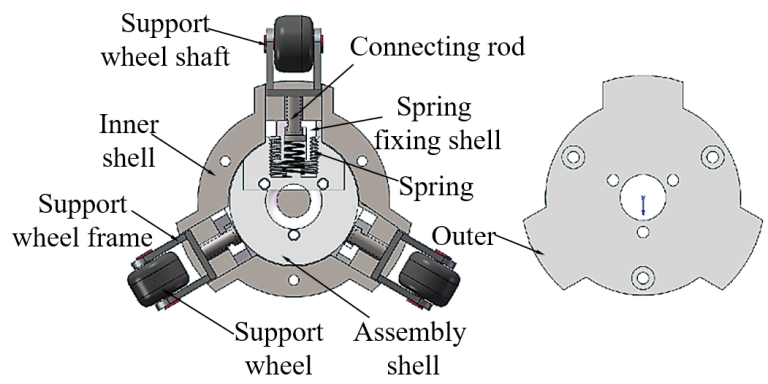

Fig. 4. Schematic diagram of support mechanism

\subsection{Leak Plugging}

The leakage plugging unit primarily comprises seven parts: an electromagnetic adsorption plate, a fixed ring, a plugging airbag, a drainage valve, a support mechanism, an airbag support ring, a plugging layer, and a repairing airbag. The overall structure is shown in Fig. 5.

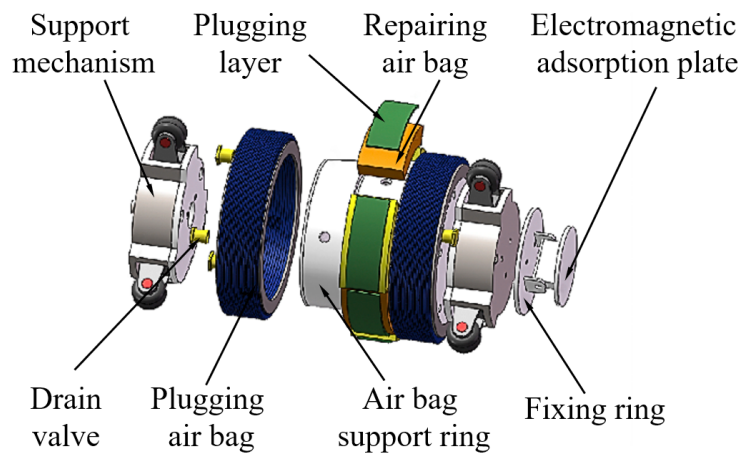

Fig. 5. Schematic diagram of the leak-plugging unit

The plugging airbag and the repairing airbag are installed on the airbag support ring, and the airbag support ring has vent holes through which the airbag can be inflated and deflated. When inflating the repairing airbag, the plugging layer connected to the outside of the airbag will be tightly pressed to the leak point of the pipeline for plugging repair work. A drainage valve is installed at both ends of the airbag. When the drainage valve is opened, the medium in the pipeline can pass through the leakage plugging unit through the drainage valve, reducing the resistance to the robot.

\section{ANALYSIS OF ROBOT MOTION CHARACTERISTICS}

\subsection{Robot Traction Analysis}

The driving unit provides power for the adaptive pipeline plugging robot. The driving mode of the driving unit is a screw drive. Assuming that the working condition of the robot is ideal, there will be no relative sliding between the driving wheel and the pipe wall, and a force analysis is performed on one of the driving wheels, as shown in Fig. 6.

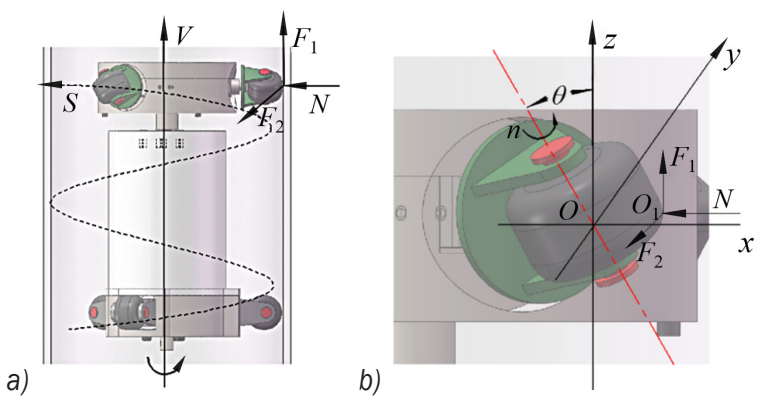

Fig. 6. Traction analysis model diagram;

a) vertical crawling analysis, and b) driving wheel force analysis

In Fig. $6 \mathrm{a}, V$ is the direction of movement of the drive unit, $S$ is the trajectory when the single wheel is in spiral operation, $N$ is the positive pressure between the pipe and the drive wheel, and $F_{1}$ is the upward friction of the driving wheel of the driving unit under the action of positive pressure. $F_{2}$ is the friction force generated by the driving wheel in the direction of the spiral movement, and $f_{1}$ is the dynamic friction coefficient; then, $F_{1}=f_{1} \cdot N$, and $\theta$ is the deflection angle of the driving wheel. When the drive unit spirals forward in the pipeline, the traction force can be expressed as Eq. (1):

$$
F=3 f_{1} \cdot N-3 F_{2} \cdot \sin \theta
$$

In Fig. 6b, the force of a single driving wheel in the pipeline is analysed, and according to the static balance equation $\sum F y=0$, Eq. (2) is obtained: 


$$
f_{2} \cdot \frac{R}{2 R} \cdot N+f_{3} \cdot \frac{r}{2 R} \cdot N-F_{2} \cdot R=0 .
$$

This is simplified as Eq. (3):

$$
F_{2}=N \cdot \frac{f_{2} \cdot R+f_{3} \cdot r}{2 R^{2}},
$$

where $f_{2}$ is the rolling friction coefficient of the driving wheel in the pipeline, $f_{3}$ is the rolling friction coefficient of the driving wheel on the axle, $r$ is the radius of the axle, and $R$ is the radius of the driving wheel.

Simultaneously solving Eqs. (1) and (3) can solve the driving force $F$, as shown in Eq. (4):

$$
F=3 N\left(f_{1}-\frac{f_{2} \cdot R+f_{3} \cdot r}{2 R^{2}} \sin \theta\right) .
$$

According to Eq. (4), when the drive unit runs in an ideal non-slippery pipe, the supporting force provided by the pipe to the drive unit is constant. In this case, the magnitude of traction force is only affected by the deflection angle of the drive wheel, and the relationship between the magnitude of traction force and the sine value of the rotation angle of the drive wheel is negatively correlated. In the range of $0^{\circ}$ to $90^{\circ}$, when the traction force needs to be increased to complete the climb or pass obstacles in the system, the driving force can be increased by reducing the deflection angle of the drive wheel. To increase the availability of the data, the angle range still needs to be tested by experiment and simulation in the subsequent process.

\subsection{Analysis of the Robot's Ability to Surmount Obstacles}

A pipeline environment is complex, and obstacles are unavoidable during the robot's operation [32] and [33]. When the adaptive plugging robot passes through obstacles, the changes of speed and force between its driving wheel and the whole robot are complex, and many factors affect its motion. Constructing obstaclecrossing equations from the main influencing factors helps to improve the robot's ability to operate in the pipeline. There are many types of obstacles in a pipeline, and a circular weld is set as the research object here in [34] to [36]. The following is a force analysis of the robot's obstacle-crossing ability under this working condition, as shown in Fig. 7.

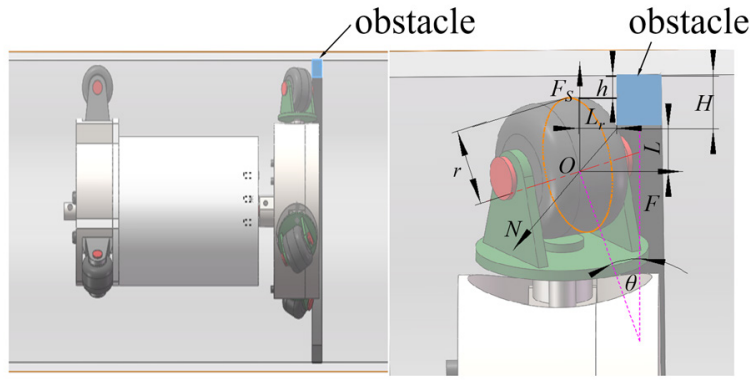

Fig. 7. Analysis diagram of driving wheel obstacle

When the robot crosses obstacles, point $O$ is assumed to be the geometric centre of the driving wheel. The convex height of the welding seam is $H$. Only when the height of the driving wheel lift is $h$ can the driving wheel pass the obstacle smoothly. When the driving wheel touches the welding seam, it will receive a reaction force $N$ given to it by the welding seam. The direction of the reaction force passes through the geometric centre of the wheel. This is the traction force $F$ required by the driving wheel to cross obstacles. The elastic force given by the variable diameter spring on the driving wheel is $F_{S}$, the radius of the driving wheel is $r$, and the deflection angle of the driving wheel when crossing the obstacle is $\theta$.

The spring force acts as resistance when the drive unit crosses the weld. To ensure that the drive unit passes by the welding seam smoothly, the relationship of Eqs. (5) to (10) must be satisfied:

$$
\begin{gathered}
M=F \cdot L \geq M_{r}=F_{s} \cdot L_{r}, \\
F=\frac{T}{3(R-h) \tan \theta}, \\
F_{s}=F_{0}+k h, \\
L=r+h-H, \\
L_{r}=\sin \theta \sqrt{r^{2}-(r+h-H)^{2}}, \\
T=\frac{9550 P}{n} .
\end{gathered}
$$

In Eq. (10), $T$ is the motor torque and $n$ the motor speed. Incorporating Eqs. (6) to (10) into Eq. (5) solves Eq. (11):

$\frac{9550 P}{3 n(R-h) \sqrt{r^{2}-(r+h-H)} \tan \theta \cdot \sin \theta} \geq \frac{F_{0}+k h}{r+h-H}$.

In Eq. (11), $P$ is the motor power, $R$ is the inner diameter of the pipe, $F_{0}$ is the spring preload, and $k$ is the spring stiffness coefficient. According to Eq. (11), during the process in which the driving unit crosses 
the welding seam, the spring is a standard part, and the spring preload and stiffness coefficient are only related to the properties of the selected spring. The parameters do not change when the attribute is determined, the pipe radius and the wheel's radius do not change, and the convex height of the welding seam remains unchanged. Under this working condition, the main factors that affect the obstacle passage of the robot are the motor torque and the deflection angle of the driving wheel.

During the obstacle-crossing operation of the drive unit, when the steering gear deflection angle is kept constant, the torque of the drive motor is reduced, and the obstacle passing performance is improved. This means that the motor speed can be reduced to increase the obstacle-crossing ability of the drive unit.

When keeping the motor speed constant, the deflection angle of the driving wheel can be adjusted to change the obstacle-crossing ability of the robot. $\tan \theta$ increases monotonically in the range of $0^{\circ}$ to $90^{\circ}$, and $\sin \theta$ also increases monotonically in the $0^{\circ}$ to $90^{\circ}$ interval, so $\tan \theta \cdot \sin \theta$ increases in this interval. Therefore, the obstacle-climbing ability of the robot can be improved to a certain extent by appropriately reducing the deflection angle of the driving wheel.

When the supporting wheel crosses the welding seam barrier, the driving wheel has already passed the welding seam. The driving wheel provides traction for the supporting wheel to pass the obstacle, and the obstacle-crossing process of the supporting wheel is analysed. The diagram for model analysis is shown in Fig. 8. When the support wheel crosses the weld seam, it will contact the weld seam. The contact point is set as $O_{1}$. The weld will give the support wheel a corresponding support force $N$. The spring has a vertical downward force $F_{\mathrm{S}}$ on the support wheel. The friction between the support wheel and the pipe is $F_{\mathrm{f}}$, and $F$ is the traction force of the robot.

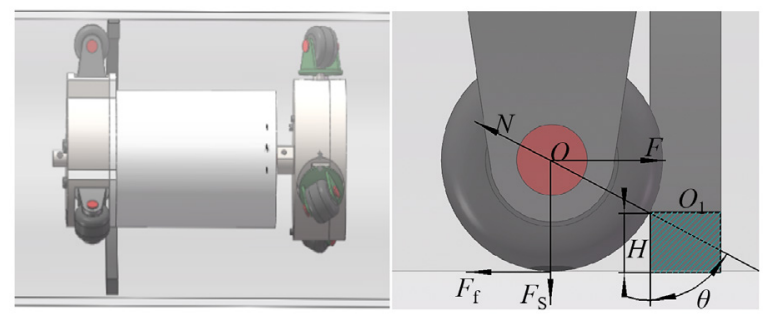

Fig. 8. Analysis diagram of the supporting wheel obstacle-crossing process

For the support wheel to cross the weld smoothly, the following conditions must be met, as shown in Eqs. (12) and (13):

$$
\begin{gathered}
\sum M\left(O_{1}\right) \geq 0, \\
F \cdot(R-H)+F_{f} \cdot H \geq F_{s} \cdot \sqrt{R^{2}-(R-H)^{2}} .
\end{gathered}
$$

When the support wheel crosses the obstacle, the friction force of the pipe on it is 0 , which is brought into Eq. (13) and simplified to obtain Eq. (14):

$$
F \geq F_{s} \frac{\sqrt{R^{2}-(R-H)^{2}}}{R-H} .
$$

According to Eq. (14), increasing the traction force can improve the robot's ability to cross the weld seam during the obstacle course of the support wheel. According to Eq. (6), when the deflection angle of the driving wheel decreases, the traction force will increase accordingly. According to Eqs. (11) and (14), when the deflection angle of the driving wheel is constant, reducing the motor speed can also increase traction.

In summary, when the robot crosses obstacles, the main factors that affect the robot's obstaclecrossing performance are motor speed and driving wheel deflection angle. During the work of the adaptive pipeline plugging repair robot, the passing performance of the robot at obstacles such as welds in the pipe can be increased by reducing the motor speed or reducing the deflection angle of the driving wheel.

\section{SIMULATION ANALYSIS OF ROBOT DRIVER UNIT}

\subsection{Simulation Analysis of the Running Speed of the Drive Unit in a Straight Pipeline}

A dynamic simulation analysis is performed with ADAMS software [37] and [38]. During the simulation, when the driving unit is running in a straight pipe, the deflection angle of the driving wheel is adjusted by the angle-adjusting steering gear, and the rotation angles of the three driving wheels need to be consistent. The selection range of the deflection angle is from $0^{\circ}$ to $90^{\circ}$, every $5^{\circ}$ is a sampling point, and the motor speed is set to $30 \mathrm{r} / \mathrm{min}$. The simulation result is shown in Fig. 9.

When the deflection angle of the driving wheel is from $5^{\circ}$ to $45^{\circ}$, the speed of the drive unit increases continuously. When the rotation angle is $45^{\circ}$, the travel speed is the highest, and the speed is $0.051 \mathrm{~m} / \mathrm{s}$. The speed increase gradually slows down from $30^{\circ}$ to $45^{\circ}$ because of the side of the driving wheel. The resistance to the pipeline will gradually increase, but the driving force is still greater than the resistance. 
When the deflection angle is from $45^{\circ}$ to $90^{\circ}$, the speed decreases continuously and is unstable. Due to the axial friction between the driving wheel and the pipe, the speed of the drive wheel gradually decreases within $45^{\circ}$ to $90^{\circ}$. In this range, the resistance is greater than the traction provided by axial motion, resulting in a decrease in speed. When it is between $15^{\circ}$ and $40^{\circ}$, the speed increases smoothly, and the running effect is the best.

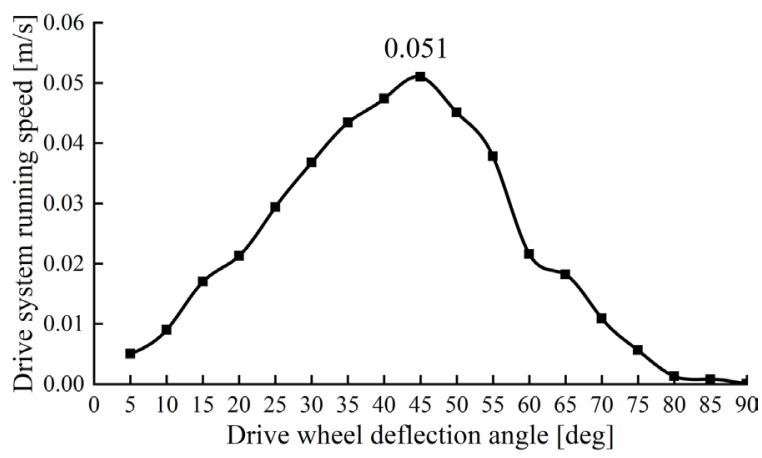

Fig. 9. Simulation value of the running speed of the drive unit in a straight pipe

To meet the design requirements, the operating speed of the robot should be within the range of $15^{\circ}$ to $40^{\circ}$, and the actual operating index can also be higher than the design index. From the speed simulation diagram of the robot running in the straight pipe, it can be seen that the running speed of the robot increases with the increase of the deflection angle within a certain range. Therefore, based on the simulation results, it is better to adjust the deflection angle of the robot driving wheel in the range of $15^{\circ}$ to $40^{\circ}$ in the actual running process to reach a better working condition.

\subsection{Simulation Analysis of the Traction Force of the Driving Unit Running in a Straight Pipeline}

In the simulation analysis of the traction force experienced by the drive unit in straight pipe operation, it can be seen from the previous section that it is better to set the deflection angle of the drive wheel from $15^{\circ}$ to $40^{\circ}$ and simulate and fit the drive wheel angle and the traction force. The simulation diagram is shown in Fig. 10.

When the driving wheel angle of the driving unit is set between $15^{\circ}$ and $45^{\circ}$, the simulation diagram shows that the relationship between the driving wheel angle and the traction force of the robot is negatively correlated. As the driving wheel angle increases, the traction force gradually decreases. The reason is that when the deflection angle increases, the axial friction force of the driving body increases, resulting in a smaller traction force along the pipe axis. When the deflection angle is $45^{\circ}$, the traction force is less than $20 \mathrm{~N}$, which does not meet the actual design requirements. Therefore, by comparing with the deflection angle range obtained by simulation, it can be seen that setting the working deflection angle of the driving unit within the range from $15^{\circ}$ to $40^{\circ}$ has the best effect.

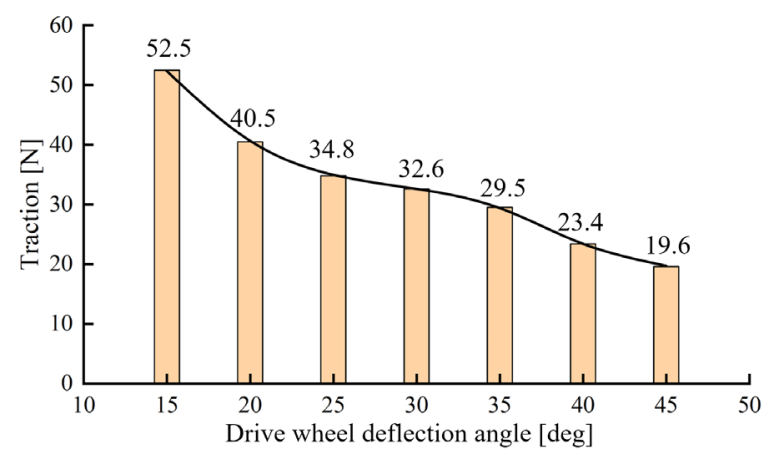

Fig. 10. Traction simulation diagram of traction robot

\subsection{Simulation Analysis of Obstacle Surmounting Stability of the Drive Unit}

In this simulation process, the weld of the pipe is set to $3 \mathrm{~mm}$. Set the speed of the spindle motor of the drive unit to 30 , and the range of deflection angle is from $15^{\circ}$ to $40^{\circ}$. Taking the centroid of the driving unit as the reference point, the stability of obstacle crossing is judged by analysing the offset displacement of the centroid on the $y$-axis. Take points every $5^{\circ}$ as a simulation value from the moment that the driving wheel contacts the weld to when the rear wheel completely leaves the weld. Starting from the driving wheel just contacting the weld, adjust the deflection angle of the driving wheel to $15^{\circ}$, and carry out a simulation to obtain the centroid offset displacement of the robot. Then, add $5^{\circ}$ each time. The deflection angles of the driving wheel are selected as $20^{\circ}, 25^{\circ}$, $30^{\circ}, 35^{\circ}$, and $40^{\circ}$ respectively for simulation. The simulation diagram is shown in Fig. 11.

Fig. 11 is analysed; Fig. 11a shows a running diagram with a deflection angle of $15^{\circ}$ and a speed of $30 \mathrm{r} / \mathrm{min}$. It can be seen that the $y$-axis displacement slowly climbs within 1 second to 1.7 seconds. In addition, Figs. 11b to f show all the crossed obstacles from 1 second to 1.5 seconds. The reason for the analysis is that under this working condition, the traction robot has too small a driving wheel deflection 


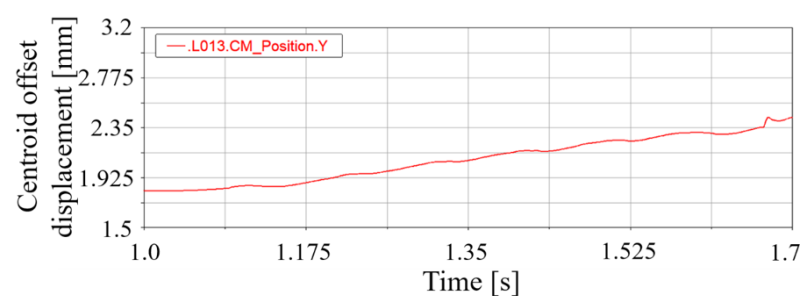

a)

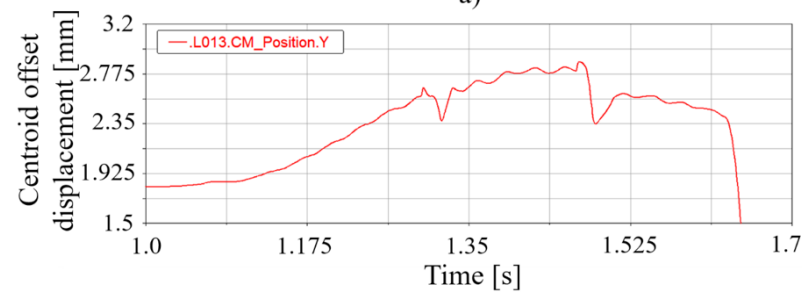

c)

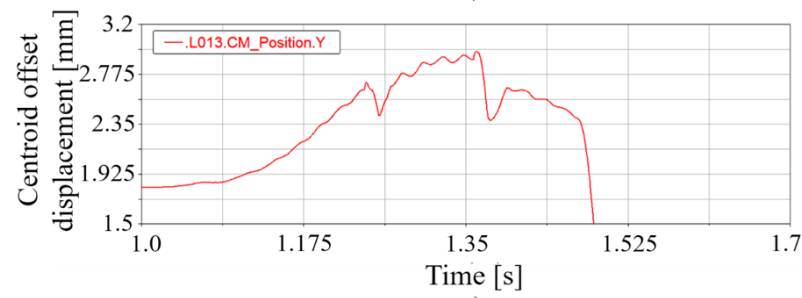

e)

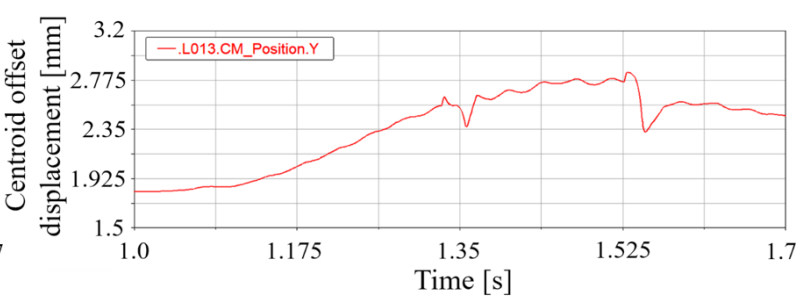

b)

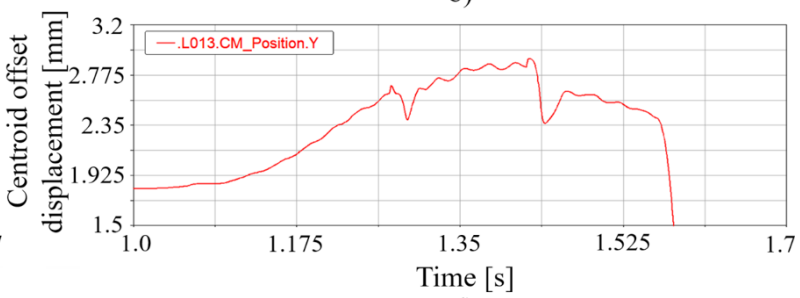

d)

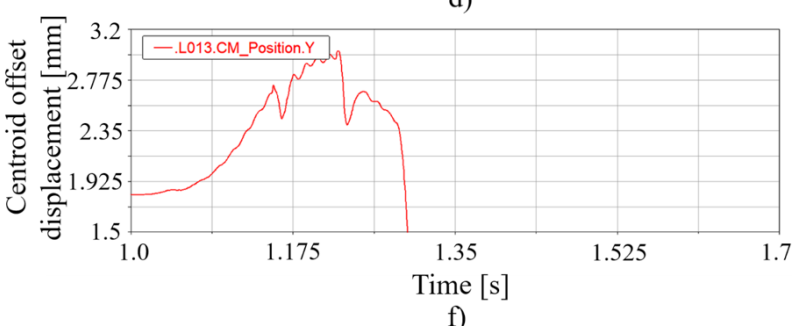

Fig. 11. Simulation diagram of obstacle-crossing stability of traction robot with different rotation angles at the same speed $30 \mathrm{r} / \mathrm{min}$; a) $15 \mathrm{deg}$, b) $20 \mathrm{deg}$, c) $25 \mathrm{deg}$, d) $30 \mathrm{deg}$, e) $35 \mathrm{deg}$, f) $40 \mathrm{deg}$

angle, the wheel rim and the welding seam have large circumferential friction, and the axial traction is small. Although this approach can continuously improve the position of the centre of mass of the robot, it can never pass the welding seam. Therefore, it can be judged that the traction robot cannot cross the welding seam when the driving wheel angle is $15^{\circ}$ and can pass the welding seam at $20^{\circ}$ to $40^{\circ}$. Then, the obstaclecrossing stability is analysed when the driving wheel angle is $20^{\circ}$ to $40^{\circ}$. It can be seen in the figure that as the deflection angle of the driving wheel increases, the fluctuation amount also increases, and the stability of obstacle crossing continues to deteriorate. When the deflection angle of the driving wheel is $20^{\circ}$, the obstacle crossing is the most stable.

\subsection{Simulation Analysis of the Maximum Obstacle Surmounting Height of the Driving Unit}

The internal environment of a pipeline is complex and diverse. When the drive unit traverses the pipeline, it may need to cross obstacles of different heights [39] and [40]. In the analysis of the previous section, we use a weld of $3 \mathrm{~mm}$ as an obstacle condition for analysis. By adjusting the deflection angle of the driving wheel, it is found that the driving unit can pass through the weld smoothly. This simulation takes a $150 \mathrm{~mm}$ pipeline as the object and sets the height of the weld to $5 \mathrm{~mm}$ for analysis to test the passability of the robot. The drive unit parameters are set as follows: the deflection angle of the drive wheel is $20^{\circ}$, and the motor speed is $30 \mathrm{r} / \mathrm{min}$. The simulation is carried out when the driving wheel crosses the $5 \mathrm{~mm}$ weld seam, and the simulation result is shown in Fig. 12.

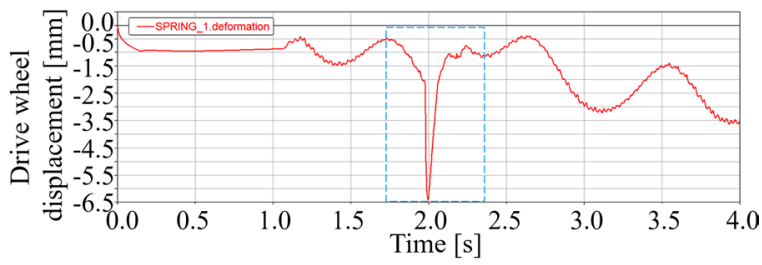

Fig. 12. Simulation diagram of driving unit by $5 \mathrm{~mm}$ weld

The process of crossing obstacles takes $1.5 \mathrm{~s}$ to $2.5 \mathrm{~s}$. At this time, the spring is compressed and contracted, and the drive wheel shifts upward. The upward displacement of the driving wheel during the obstacle course is approximately $5.5 \mathrm{~mm}$. Because the robot can be designed to meet the diameter change in the range of $20 \mathrm{~mm}$, the drive unit can pass the 5 $\mathrm{mm}$ weld under this working condition to meet the design requirements. Second, under the condition that 


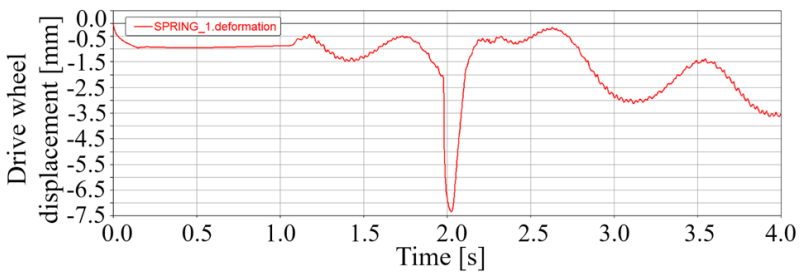

a)

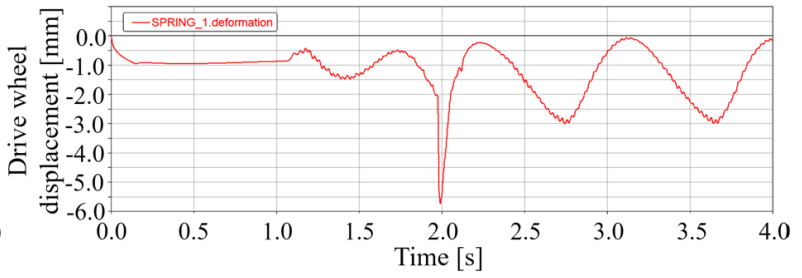

b)

Fig. 13. Simulation diagram of the driving unit through a) $6 \mathrm{~mm}$, and b) $7 \mathrm{~mm}$ welds

the parameter setting is unchanged and the working conditions are the same, the weld height is set to $6 \mathrm{~mm}$ and $7 \mathrm{~mm}$. Under these two conditions, a simulation analysis of the drive unit's passability is performed. The simulation diagram is shown in Fig. 13.

It can be seen in Fig. 13a that when the drive unit passes through the $6 \mathrm{~mm}$ weld seam, the maximum displacement of the drive wheel is $6.5 \mathrm{~mm}$. Therefore, the drive unit can pass the $6 \mathrm{~mm}$ weld seam smoothly. It can be seen in Fig. 13b that when the drive unit passes through the $7 \mathrm{~mm}$ weld seam, the maximum offset displacement cannot exceed $7 \mathrm{~mm}$. Combined with the simulation, it can be seen that the $7 \mathrm{~mm}$ weld seam jams the drive unit driving wheel, and the drive body continues to rotate after being jammed, causing the displacement of the driving wheel to perform periodic motion under the action of spring force and gravity.

From the above analysis, it can be concluded that the maximum obstacle-crossing height that the drive unit can pass smoothly is $6 \mathrm{~mm}$, which meets the design requirements.

\section{EXPERIMENTAL VERIFICATION}

A prototype of an adaptive plugging robot for buried pipeline leakage is manufactured and assembled in full accordance with the above design requirements, as shown in Fig. 14.

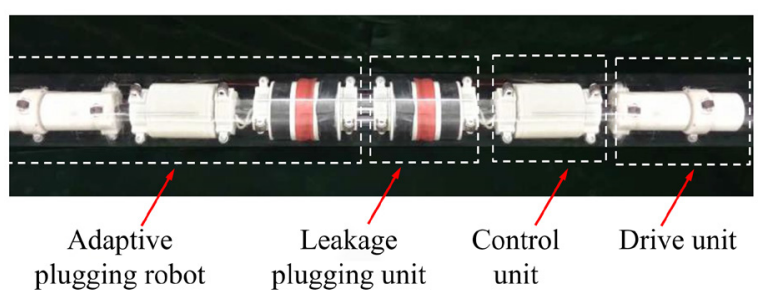

Fig. 14. Prototype of an adaptive plugging robot for buried pipeline leakage

The mass of the driving unit was measured to obtain $1.3 \mathrm{~kg}$, the total mass of a single set of adaptive plugging robots was $3.5 \mathrm{~kg}$, the overall mass of the two sets of robots was $7 \mathrm{~kg}$, the length of the drive unit was $230 \mathrm{~mm}$, the length of a single adaptive plugging robot was $765 \mathrm{~mm}$, and the length of the combined robot was $1530 \mathrm{~mm}$. The control unit mainly controls the steering gear of the driving wheel, the motor, the drainage valve, the airbag deflation valve and the airbag inflator.

The results of a speed test for the prototype are shown in Fig. 15. It can be seen in the figure that the running speed curve of the prototype obtained during the test is roughly the same as the curve of the simulation analysis, but the result of the simulation analysis is always greater than the experimental result.

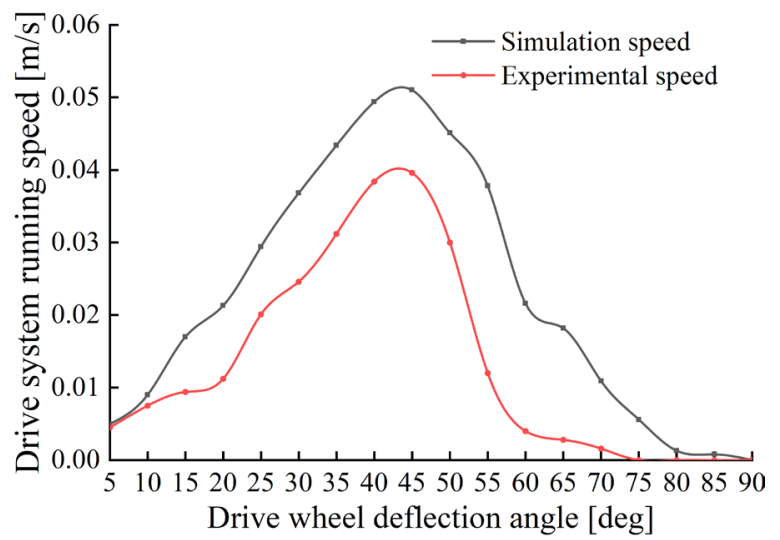

Fig. 15. Comparison of running speed of test prototype

It can be seen from the operating speed comparison chart that when the deflection angle is between $5^{\circ}$ and $45^{\circ}$, the deflection angle of the drive wheel gradually increases, and the speed of the prototype also increases. The speed of the prototype reaches its peak at $45^{\circ}$, and the peak speed is approximately $0.039 \mathrm{~m} / \mathrm{s}$. This result also meets the design requirements.

When testing the traction force of the prototype, it is necessary to use a spring dynamometer for measurement. The spindle speed of the drive unit is set to $30 \mathrm{r} / \mathrm{min}$, the drive wheel angle is set to $15^{\circ}$ to $45^{\circ}$ 
in turn, the system is started, and the drive unit drags the leakage plugging unit and the control unit forward until it is completely pulled by the dynamometer, which reads the dynamometer and indicates the number. The comparison between the experimental result and the simulation is shown in Fig. 16.

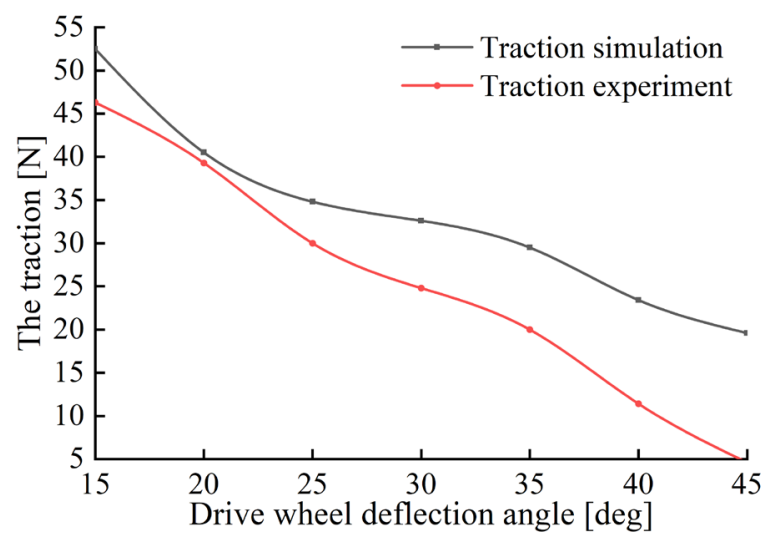

Fig. 16. Comparison diagram of driving force test of prototype

Comparing the simulation results with the experimental results, it can be concluded that the trends of the two curves are roughly the same, which proves the correctness of the simulation results. It can be seen in Fig. 16. that the experimental value is always lower than the simulation value. The reason for this phenomenon is that the simulation is moving under ideal conditions, and the design and installation of the experimental prototype and the experimental environment will have certain accuracy errors. After the deflection angle of the drive wheel is greater than $20^{\circ}$, the gap between the simulation results and the experimental results is larger than before, mainly because as the speed continues to increase, the impact of the assembly accuracy of the drive system on the operating state gradually appears. When the deflection angle is $35^{\circ}$, the traction force is $20 \mathrm{~N}$, which is the most basic requirement of the design. In the simulation analysis, $20 \mathrm{~N}$ is reached at $40^{\circ}$. The assembly accuracy of the prototype has a significant impact on its specific operating state. Therefore, based on the original simulation conclusions, the upper limit of deflection angle during stable operation of the system shall be further reduced from $40^{\circ}$ to $35^{\circ}$. The lower limit of deflection angle during stable operation of the system is $20^{\circ}$.

The obstacle-crossing ability test of the prototype adopts the driving unit test method. The obstaclecrossing ability test is carried out in a $2000 \mathrm{~mm}$ straight pipe. Several rubber sealing rings are bound and bonded on the inner wall of the pipeline to simulate obstacles, and the main protrusion heights are $5 \mathrm{~mm}, 6 \mathrm{~mm}$, and $7 \mathrm{~mm}$, respectively. Because the friction coefficient between the outer surface of the rubber seal ring and the inner wall of the experimental pipe is almost the same, the simulation as an obstacle is close to the real obstacle. The simulation results show that the drive unit can pass by $6 \mathrm{~mm}$ welds, so the test directly simulates $5 \mathrm{~mm}, 6 \mathrm{~mm}$, and $7 \mathrm{~mm}$ welds to test the possibility of the prototype. The experimental environment is shown in Fig. 17.

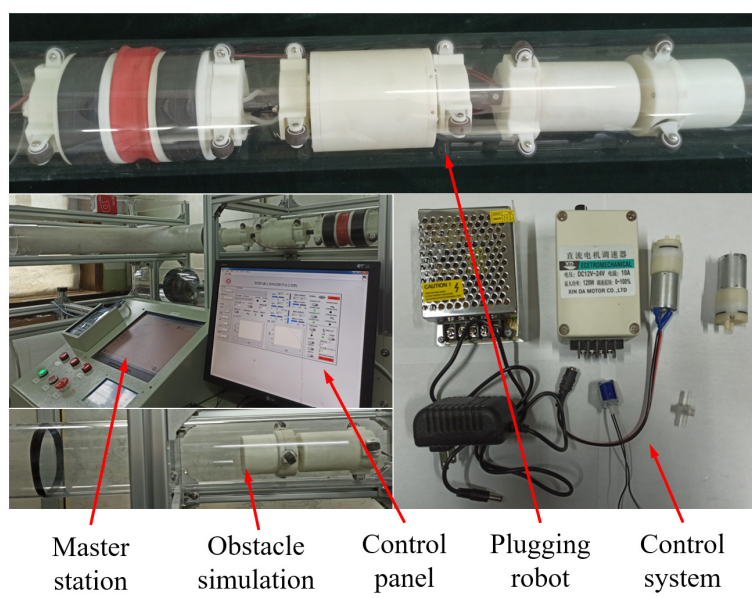

Fig. 17. Test diagram of the obstacle-crossing ability of the test prototype

The results of the prototype's obstacle-crossing capability are shown in Table 2.

An analysis of Table 2. shows that when the driving wheel rotation angle is set to $15^{\circ}$, the angle formed between the outer surface of the driving wheel and the obstacle is too small, which results in slipping and an inability to cross the obstacle. Therefore, $20^{\circ}$ is the minimum passing angle when the driving wheel crosses the obstacle, which is consistent with the simulation results. When the driving wheel angle of the prototype is $20^{\circ}$, it can pass $6 \mathrm{~mm}$ obstacles but cannot pass $7 \mathrm{~mm}$ obstacles.

Table 2. Obstacle clearance results for prototype

\begin{tabular}{|c|c|c|c|}
\hline Angle Height & $5 \mathrm{~mm}$ & $6 \mathrm{~mm}$ & $7 \mathrm{~mm}$ \\
\hline $15^{\circ}$ & Unpassable & Unpassable & Unpassable \\
\hline $20^{\circ}$ & Accessible & Accessible & Unpassable \\
\hline $25^{\circ}$ & Accessible & Accessible & Unpassable \\
\hline $30^{\circ}$ & Accessible & $\begin{array}{c}\text { Pass after } \\
\text { multiple skidding }\end{array}$ & Unpassable \\
\hline $35^{\circ}$ & Accessible & Unpassable & Unpassable \\
\hline
\end{tabular}

Although $25^{\circ}$ can pass $6 \mathrm{~mm}$ obstacles, the exercise state is not good and cannot pass $7 \mathrm{~mm}$ 
obstacles. At $30^{\circ}$, the drive unit has difficulty passing the $6 \mathrm{~mm}$ obstacle. When the running speed increases, the manufacturing accuracy and installation accuracy of parts are low due to the assembly process of the prototype. The vibration and centroid fluctuation of the prototype robot for adaptive plugging and repairing pipeline are large, which reduce the ability to overcome obstacles. The camera shakes more severely at $35^{\circ}$ and cannot pass $6 \mathrm{~mm}$ obstacles.

In the simulation results, when the prototype passes through the welding seam, the driving wheel deflection angle is set to $20^{\circ}$, and when the motor speed is $30 \mathrm{r} / \mathrm{min}$, the maximum obstacle height that can be passed is $6 \mathrm{~mm}$. The experiment is consistent with the simulation results.

\section{CONCLUSIONS}

(1) To meet the design requirements, the robot uses a multi-body distributed structure in which each unit is connected in series. The main body connects two sets of adaptive plugging robots with electromagnetic adsorption modules. The self-adaptive plugging robot is composed of a drive unit, a control unit, and a leak-plugging unit, and each unit is connected by a parallel coupling. The drive unit adopts the driving form of a motor drive and spiral travel, incorporates the expansion and contraction of the spring to match the diameter, and uses a corrugated airbag to complete the plugging.

(2) Through an analysis of the obstacle-crossing mechanism of the robot, it is concluded that reducing the rotational speed of the motor or reducing the deflection angle of the driving wheel can increase the passing performance of the robot at obstacles such as welds in the pipe.

(3) A prototype is assembled, and related experiments are conducted to determine that the overall structure of the prototype meets the design requirements. The traction force and obstaclecrossing ability of the prototype are verified experimentally. The results show that the running index meets the design requirements when the driving wheel deflection angle is set at $20^{\circ}$ to $35^{\circ}$. The deflection angle of the driving wheel is in this range; the smaller the angle is, the better the running effect is, and the best running angle is $20^{\circ}$. Under the best operating conditions, the maximum obstacle height of the prototype is 6 $\mathrm{mm}$.

(4) The design of the buried pipeline leakage selfadaptive plugging robot provides an important reference for the research and development of equipment in the field of pipeline leakage emergency prevention and control.

\section{ACKNOWLEDGEMENTS}

The work described in this paper was supported by a research project supported by the Shanxi Scholarship Council of China (2020-110), the Fundamental Research Program of Shanxi Province (20210302123038) and the National Natural Science Foundation of China (52174147). The authors would like to express their gratitude for the support of this study. Moreover, the authors sincerely thanks Professor Wu Wenge of North University of China for his critical discussion and Dr. Dahua Xie of Silicon Valley USA for linguistic assistance during the preparation of this manuscript. At the same time, we accepted the professional language editing service of American Journal Experts (AJE).

\section{REFERENCES}

[1] Witkowski, A., Rusin, A., Majkut, M., Stolecka, K. (2018). Analysis of compression and transport of the methane/ hydrogen mixture in existing natural gas pipelines. International Journal of Pressure Vessels and Piping, vol. 166, p. 24-34, D0I:10.1016/j.ijpvp.2018.08.002.

[2] Zeng, J., Sun, C.X., Zhu, Z.J., Wu, J.L., Chen H.S. (2018). Uncertainty analysis for natural gas transport pipeline network layout: a new methodology based on Monte Carlo method. Journal of Advanced Transportation, vol. 2018, art. ID 9213648, DOI:10.1155/2018/9213648.

[3] Li, M., Liu, L.L., Li, Y., Xu Y.C. (2018). Evaluating the risk of natural gas pipeline operation management in intuitionistic fuzzy linguistic environments. Mathematical Problems in Engineering, vol. 2018, art. ID 3960496, DOI:10.1155/2018/3960496.

[4] Vasseghi, A., Haghshenas, E., Soroushian, A., Rakhshandeh, M. (2020). Failure analysis of a natural gas pipeline subjected to landslide. Engineering Failure Analysis, vol. 119, art. ID 105009, DOI:10.1016/J.engfailanal.2020.105009.

[5] Deng, Y.J., Hu, H.B., Yu, B., Sun, D.L., Hou, L., Liang, Y.T. (2017). A method for simulating the release of natural gas from the rupture of high-pressure pipelines in any terrain. Journal of Hazardous Materials, vol. 342, p. 418-428, D0l:10.1016/j. jhazmat.2017.08.053.

[6] Mahmoodzadeh, Z., Wu, K.Y., Droguett, E.L., Mosleh, A. (2020). Condition-Based maintenance with reinforcement learning for dry gas pipeline subject to internal corrosion. Sensors, vol. 20, no. 19, art. ID 5708, D0I:10.3390/s20195708.

[7] Dong, F.F., Bie, X.M., Tian, J.P., Xie, X.D., Du, GF. (2019). Experimental and numerical study on the strain behavior of buried pipelines subjected to an impact load. Applied Sciences, vol. 19, no. 16, art. ID 3284, D0l:10.3390/ app9163284. 
[8] Zhang, D.F., Bie, X.M., Zeng, X., Lei, Z., Du, G.F. (2020). Experimental and numerical studies on mechanical behavior of buried pipelines crossing faults. Structural Engineering and Mechanics, vol. 75, no. 1, p. 71-89, Dol:10.12989/ sem.2020.75.1.071.

[9] Liang, Z.D., Jeng, D.S., Liu, J.W. (2020). Combined wavecurrent induced seabed liquefaction around buried pipelines: Design of a trench layer. Ocean Engineering, vol. 212, art. ID 107764, DOl:10.1016/j.oceaneng.2020.107764.

[10] Yan, H.W., Wang, L., Li, P.C., Wang, Z.J., Yang, X., Hou, X.R. (2020). Research on passing ability and climbing performance of pipeline plugging robots in curved pipelines. IEEE Access, vol. 8, p. 173666-173680, D0l:10.1109/ ACCESS.2020.3025560.

[11] Wang, W.M., Liu, X., Wang, H., Fan, J.C., Zhu, X.X. (2018). Pose overshoot analysis of the marine spherical double plugging in twisting process. Proceedings of The Institution of Mechanical Engineers Part M-Journal of Engineering for The Maritime Environment, vol. 232, no. 2, p. 245-252, DOI:10.1177/1475090217722307.

[12] Cheng, F., Liu, J.P., Wang, J., Yang, Z.D., Abbasi S.S., Tian, Z.J., Xu. H. (2018). Locating leaking buried pipes based on ground micro seismic records in 3D space. Surveys in Geophysics, vol. 39, no. 5, p. 993-1007, D0l:10.1007/s10712-018-9479-x.

[13] Kakogawa, A., Nishimura, T., Ma, SG. (2016). Designing arm length of a screw drive in-pipe robot for climbing vertically positioned bent pipes. ROBOTICA, vol. 34, no. 2, p.306-327, DOI:10.1017/S026357471400143X.

[14] Kim, K.T., Kim, H.W., Sik, K.Y., Chang H.Y., Bu, T.B., Park, H. (2015). Real-time corrosion control system for cathodic protection of buried pipes for nuclear power plant. Corrosion Science and Technology, vol. 14, no. 1, p. 12-18, DOI:10.14773/cst.2015.14.1.12.

[15] Zhang, C.Y., Chen, G.Z., Miao, C.W., Tang, Y.J., Bai, N. (2020). Driving pulsation analysis of electro-hydraulic robot. Journal of Advanced Mechanical Design Systems and Manufacturing, vol. 14, no. 1, D0l:10.1299/jamdsm.2020jamdsm0012.

[16] Han, M., Zhao, F., San D., Zhai K.D., Wang D., Zhou, J. (2017). Structural design and driving force analysis of a nuclear pipeline robot. Electromechanical Engineering, vol. 34, no. 07, p. 696-701, D0l:10.3969/j.issn.1001.4551.2017.07.002.

[17] Zhao, W., Kamezaki, M., Yoshida, K., Yamaguchi, K., Konno, M., Onuki, A., Sugano, S. (2019). A coordinated wheeled gas pipeline robot chain system based on visible light relay communication and illuminance assessment. Sensors, vol. 19, no. 10, art. ID 2322, D0l:10.3390/s19102322.

[18] Liu Q.Y., Li, Y.J., Ren, T., Chen, Y.H. (2014). Active screw driven pipeline robot. Robot, vol. 36, no. 6, p. 711-718, DOl:10.13973/j.cnki.robot.2014.0711.

[19] Tu, Q., Liu, Q.Y., Ren, T., Li, Y.J. (2019). Obstacle crossing and traction performance of active and passive screw pipeline robots. Journal of Mechanical Science and Technology, vol. 33, no. 5, p. 2417-2427, D0l:10.1007/s12206-019-0440-9.

[20] Li, P., Tang, M., Lyu, C.Y., Fang, M.J., Duan, X.G., Liu, Y.H. (2018). Design and analysis of a novel active screw-drive pipe robot. Advances in Mechanical Engineering, vol. 10, no. 10, DOI:10.1177/1687814018801384.
[21] Kakogawa, A., Nishimura, T., Ma, S. (2016). Designing arm length of a screw drive in-pipe robot for climbing vertically positioned bent pipes. Robotica, vol. 34, no. 2, p. 306-327, DOI:10.1017/S026357471400143X.

[22] Nishimura, T., Kakogawa, A., Ma, S. (2013). Improvement of a screw drive in-pipe robot with pathway selection mechanism to pass through T-branches. Journal of Robotics and Mechatronics, vol. 25, no. 2, p. 340-346, D0l:10.20965/ jrm.2013.p0340.

[23] Wang, Y.F., Pandit, P., Kandhari, A., Liu, Z.H., Daltorio, K.A. (2020). Rapidly exploring random tree algorithm-based path planning for worm-like robot. Biomimetics, vol. 5, no. 2, art. ID 26, D0l:10.3390/biomimetics5020026.

[24] Jeon, W., Kim, I., Park, J., Yang, H. (2013). Design and control method for a high-mobility in-pipe robot with flexible links. The Industrial Robot, vol. 40, no. 3, p. 261-274, DOl:10.1108/01439911311309960.

[25] Lu, X.J., Wang, K., Hu, T.T. (2020). Development of an annelidlike peristaltic crawling soft robot using dielectric elastomer actuators. Bioinspiration \& Biomimetics, vol. 15, no. 4, DOI:10.1088/1748-3190/ab8af6.

[26] Mishra, D., Yadav, R.S., Agrawal, K.K. (2020). Kinematic modelling and emulation of robot for traversing over the pipeline in the refinery. Microsystem Technologies, vol. 26, no. 3, p. 1011-1020, D0l:10.1007/s00542-019-04615-9.

[27] Zagler, A., Pfeiffer, F. (2003). "MORITZ" a pipe crawler for tube junctions. Proceedings - IEEE International Conference on Robotics and Automation, vol. 3, p. 2954-2959, D0l:10.1109/ ROBOT.2003.1242044.

[28] Aleksandersen, J., Tveit, E. (2001). The smart plug: A remotely controlled pipeline isolation system. Proceedings of the International Offshore and Polar Engineering Conference, vol. 2, p. 192-199.

[29] Yan, H.W., Yang, X., Hou, X.R., Wang, L., Li, P.C. (2020). Research on the performance of a new external occlusion device for pressure vessel leakage. Strojniški vestnik - Journal of Mechanical Engineering, vol. 66, no. 11, p. 668-676, DOI:10.5545/sv-jme.2020.6810.

[30] Bowie, A. (2012). A comparison of double block and bleed technologies. Proceedings of the Biennial International Pipeline Conference, vol 1, p. 425-439, p. 9780791845127 , DOI: 10.1115/IPC2012-90020.

[31] An, Y., Wang, X.C., Yue, B., Qu, Z.G., Wu, L.Q., Chu, R.H. (2019). Compensation of sound velocity variation based on resampling algorithm for natural gas pipeline safety monitoring. Measurement, vol. 148, D0l:10.1016/j. measurement.2019.106942.

[32] Zhang, H.W., Zhang, S.T., Wang, Y.H., Liu, Y.H., Yang, Y.N., Zhou, T., Bian, H.Y. (2021). Subsea pipeline leak inspection by autonomous underwater vehicle. Applied Ocean Research, vol. 107, art. ID 102321, D0I:10.1016/j.apor.2020.102321.

[33] Cao, Y., Suzuki, S., Hoshino, Y. (2016). Uphill and level walking of a three-dimensional biped quasi-passive walking robot by torso control. Robotica, vol. 34, no. 3, p. 483-496, D0I:10.1017/S0263574714001593.

[34] Du, Q.L., Lu, X.P., Wang, Y.K., Liu, S.A. (2020). The obstaclesurmounting analysis of a pole-climbing robot. International 
Journal of Advanced Robotic Systems, vol. 17, no. 6, DOI:10.1177/1729881420979146.

[35] Xi, F.Y., Jiang, Q.S. (2019). Dynamic obstacle-surmounting analysis of a bilateral-wheeled cable-climbing robot for cablestayed bridges. Industrial Robot: The International Journal of Robotics Research and Application, vol. 46, no. 3, p. 431-443, D0I:10.1108/IR-07-2018-0152.

[36] Zhu, X.X., Wang, W., Zhang, S.M., Liu, S.H. (2017). Experimental research on the frictional resistance of fluid-driven pipeline robot with small size in gas pipeline. Tribology Letters, vol. 65, no. 2, Dol:10.1007/s11249-017-0830-z.

[37] Korayem, A.H., Irani, M., Babaee, H., Korayem, M.H. (2017). Maximum load of flexible joint manipulators using nonlinear controllers. Robotica, vol. 35, no. 1, p. 119-142, D0l:10.1017/ S0263574715000028.
[38] Korayem, M.H., Dehkordi, S.F. (2018). Derivation of motion equation for mobile manipulator with viscoelastic links and revolute-prismatic flexible joints via recursive Gibbs-Appell formulations. Robotics and Autonomous Systems, vol. 103, p. 175-198, D0I:10.1016/j.robot.2018.02.013.

[39] Li, H., Li, R.Q., Zhang, J.W., Zhang, P.Y. (2020). Development of a pipeline inspection robot for the standard oil pipeline of China national petroleum corporation. Applied Sciences, vol. 10, no. 8, DOl:10.3390/app10082853.

[40] Ovalle-Magallanes, E., Aldana-Murillo, N.G., Avina-Cervantes, J.G., Ruiz-Pinales, J., Cepeda-Negrete, J., Ledesma, S. (2021). Transfer learning for humanoid robot appearance-based localization in a visual map. IEEE Access, vol. 9, p. 68686877, DOI:10.1109/ACCESS.2020.3048936. 